ISSN 2179-6750

\title{
Qualidade de vida do portador de hipertensão arterial sistêmica assistido por uma estratégia de saúde da família de Minas Gerais
}

Quality of life of patients with systemic arterial hypertension assisted by a family health strategy of Minas Gerais

Cualidad de vida del portador de hipertensión arterial sistémica asistido por una estrategia de salud de la familia de Minas Gerais

Patrick Leonardo Nogueira da SILVA ${ }^{(1)}$

Daniele Cristina MOREIRA ${ }^{(1)}$

Aurelina Gomes e MARTINS ${ }^{(1)}$

Elaine Cristina Santos ALVES ${ }^{(1)}$

${ }^{(1)}$ Enfermeiro(a), Universidade Estadual de Montes Claros - UNIMONTES, Montes Claros, MG, Brasil.

Recebido: 24 fev 2017

Revisado: 10 maio 2017

Aceito: 28 jul 2017

Autor de

correspondência:

Patrick Leonardo Nogueira

da Silva

patrick_mocesp70@hotmail.com

Conflito de interesses: Os autores declaram não haver nenhum interesse profissional ou pessoal que possa gerar conflito de interesses em relação a este manuscrito.

\begin{abstract}
Resumo
A hipertensão arterial sistêmica é caracterizada por níveis elevados e sustentados da pressão arterial na qual se configura um dos principais problemas de saúde pública por se tratar de uma doença crônica, multifatorial e com alto potencial de risco para doenças cardiovasculares, cerebrovasculares e renais. Este estudo objetivou avaliar a qualidade de vida do portador de hipertensão arterial sistêmica assistido por uma Estratégia de Saúde da Família de Minas Gerais. Trata-se de um estudo descritivo, exploratório, transversal, com abordagem quantitativa, realizado com 253 hipertensos cadastrados em uma unidade básica de saúde. Utilizou-se um questionário semiestruturado, adaptado e validado na qual os dados foram apresentados em tabelas. $O$ tratamento dos dados se deu através de análise estatística descritiva simples e análise de correlação empregandose o teste qui-quadrado. Observou-se maior parte feminina na qual relataram que a hipertensão não afeta a qualidade de vida. $O$ principal fator de interferência na qualidade de vida foi a ingestão de sal (valor $p=0,05$ ), o que demonstrou a importância de se controlar o consumo do mesmo para o controle da pressão arterial e para a prevenção de complicações. A percepção sobre qualidade de vida não se mostrou associada à condição crônica e sim com a postura do indivíduo frente à doença e às mudanças impostas no estilo de vida. O reconhecimento da gravidade da doença e a possibilidade do desenvolvimento de complicações podem ser utilizados pela equipe de saúde como ferramentas auxiliares na educação para a adoção de hábitos de vida saudáveis.
\end{abstract}

Descritores: Hipertensão; Qualidade de Vida; Atenção Primária à Saúde. 


\begin{abstract}
Systemic arterial hypertension is characterized by elevated and sustained levels of blood pressure, which is one of the main public health problems because it is a chronic, multifactorial disease with a high risk potential for cardiovascular, cerebrovascular and renal diseases. This study aimed to evaluate the quality of life of patients with systemic arterial hypertension assisted by a Family Health Strategy of Minas Gerais. This is a descriptive, exploratory, cross-sectional study with a quantitative approach, performed with 253 hypertensive patients enrolled in a basic health unit. We used a semi-structured, adapted and validated questionnaire in which the data were presented in tables. Data were processed through simple descriptive statistical analysis and correlation analysis using the chi-square test. It was observed a greater female part in which they reported that the hypertension does not affect the quality of life. The main factor of interference in quality of life was salt intake ( $p$ value $=0.05$ ), which demonstrated the importance of controlling the consumption of salt for the control of blood pressure and for the prevention of complications. The perception about quality of life was not associated with the chronic condition, but with the attitude of the individual to the disease and the changes imposed on the lifestyle. The recognition of the severity of the disease and the possibility of developing complications can be used by the health team as auxiliary tools in education for the adoption of healthy living habits.
\end{abstract}

Keywords: Hypertension; Quality of Life; Primary Health Care.

\section{Resumen}

La hipertensión se caracteriza por niveles altos y sostenidos de la presión arterial en el que se configura un importante problema de salud pública debido a que es una enfermedad crónica y multifactorial con alto riesgo potencial de enfermedades cardiovasculares, cerebrovasculares y enfermedades renales. Este estudio tuvo como objetivo evaluar la calidad de vida de soporte de la hipertensión con la asistencia de una estrategia de salud para la familia de Minas Gerais. Se trata de un estudio descriptivo, exploratorio, cruce con un enfoque cuantitativo, realizado con 253 pacientes hipertensos inscritos en una unidad básica de salud. Se utilizó un cuestionario semi-estructurado, adaptado y validado en el que se presentaron los datos en las tablas. El análisis de datos se realizó mediante el análisis estadístico descriptivo simple y análisis de correlación utilizando la prueba de chi-cuadrado. Una parte hembra superior en el que informó que la hipertensión no afecta a la calidad de vida. El factor de interferencia principal en la calidad de vida fue la ingesta de sal $(p=$ 0,05), lo que demuestra la importancia de controlar el mismo consumo para el control de la presión arterial y la prevención de complicaciones. La percepción de la calidad de vida no se asoció con la condición crónica, pero con la actitud del individuo hacia la enfermedad y los cambios impuestos en el estilo de vida. El reconocimiento de la gravedad de la enfermedad y la posibilidad de desarrollar complicaciones puede ser utilizado por el equipo de salud como herramientas auxiliares en la educación a la adopción de hábitos de vida saludables.
\end{abstract}

Palabras-claves: Hipertensión; Calidad de Vida; Atención Primaria de Salud.

\title{
Introdução
}

Nas últimas décadas, observou-se uma mudança no perfil de morbimortalidade no Brasil e no mundo, o que indica um aumento das doenças crônico-degenerativas. Os avanços nos tratamentos o que possibilitou um controle mais efetivo dessas enfermidades com consequente aumento da sobrevida das pessoas acometidas, entre elas os portadores de hipertensão arterial sistêmica (HAS) são notórios. ${ }^{1}$ A HAS é uma condição clínica 
multifatorial caracterizada por níveis elevados e sustentados da pressão arterial (PA), em que a pressão arterial sistólica (PAS) se encontra acima de $140 \mathrm{mmHg}$, e a pressão arterial diastólica (PAD) acima de $90 \mathrm{mmHg}{ }^{2}$

Configura-se como um dos principais problemas de saúde pública por se tratar de uma doença crônica, multifatorial e com alto potencial de risco para doenças cardiovasculares (DCV), cerebrovasculares e renais. A HAS é um grave problema de saúde pública no Brasil e no mundo. Sua prevalência no Brasil varia entre 22-44\% para adultos (32\% em média), identificando a mais de 50\% para indivíduos com 60-69 anos e 75\% em pessoas com mais de 70 anos. $^{3}$

Doença crônica é a enfermidade que tem uma história natural prolongada, caracterizada pela multiplicidade de fatores associados, tais como: fatores etiológicos; longo período de latência e longo curso assintomático; evolução clínica lenta, prolongada e permanente; além da evolução para complicações. A doença crônica traz para a vida do cliente uma série de transformações, inclusive ligadas ao autoconceito, em função de sua possibilidade de agravo e dificuldade de aceitação e adaptação à sua nova condição, podendo acarretar sintomas de depressão e ansiedade. Os objetivos do tratamento das doenças crônicas são diminuir a morbimortalidade e manter a qualidade de vida (QV) dos indivíduos doentes. ${ }^{4}$

Entre as doenças crônicas, destaca-se a HAS a qual é responsável, em todo o mundo, por elevadas taxas de incapacidade física e mortalidade além da interferência na QV dos indivíduos acometidos, o que gera uma grande preocupação nos trabalhadores do setor saúde e, por conseguinte, estimula estudiosos em distintas áreas do saber a desenvolverem pesquisas tendo como foco este problema. ${ }^{5}$

Avaliar a QV é estimar o impacto físico e psicossocial que as enfermidades, disfunções ou incapacidades acarretam para as pessoas acometidas. Estudos relacionados à QV, especialmente em relação às doenças crônicas, proporcionam esclarecimento sobre o impacto da patologia com relação às ações cotidianas; discussões que possibilitam 
abordar as diferentes terapêuticas; a melhora na assistência e reabilitação e levar em consideração a opinião do cliente acerca da sua preferência em relação ao tratamento estabelecido. ${ }^{6}$ Sendo assim, objetivou-se avaliar a QV do portador de HAS assistido por uma ESF de Minas Gerais.

\section{Metodologia}

Artigo da monografia intitulada "Qualidade de vida de hipertensos assistidos pela Estratégia de Saúde da Família", apresentada ao Departamento de Enfermagem da Universidade Estadual de Montes Claros - UNIMONTES, Montes Claros (MG), Brasil, 2013.

Trata-se de um estudo descritivo, exploratório, transversal, com abordagem quantitativa, realizado na Estratégia Saúde da Família - ESF do bairro Morrinhos II localizado no município de Montes Claros, MG. A ESF apresenta 366 indivíduos inscritos no Programa de Hipertensão e Diabetes - HIPERDIA). Deste total, apenas 253 hipertensos $(69,1 \%)$ cadastrados na área de abrangência compuseram a amostra.

Foram adotados os seguintes critérios de inclusão para participação no estudo: estar cadastrado no HIPERDIA há pelo menos seis meses e participar das atividades desenvolvidas; ser maior de 18 anos; ter diagnóstico médico apenas de HAS; ser encontrado em até no máximo três tentativas para a realização da coleta dos dados.

O instrumento de coleta de dados foi o "Mini-Questionário de QV em Hipertensão Arterial - MINICHAL" que consiste em um questionário fechado, anônimo, validado e adaptado. $^{7}$ Também foi utilizado um questionário de elaboração própria, com aspectos relevantes para a pesquisa, abordando variáveis socioeconômicas, demográficas e clínicas, na qual foi realizado um pré-teste numa população semelhante à da pesquisa selecionada especificamente para essa atividade de modo a validar o instrumento de coleta de dados. Este possibilitou estimar o tempo necessário para a aplicação dos questionários, bem como das medidas antropométricas e aferição da PA. 
Dessa forma, a coleta de dados foi realizada no período compreendido entre julho a setembro de 2013, durante o funcionamento da Unidade Básica de Saúde (UBS), em especial, nas datas de realização das reuniões educativas, como as do HIPERDIA, bem como reunião com idosos e em visitas domiciliares, previamente informadas à instituição. Em seguida, foram avaliados os dados antropométricos (Índice de Massa Corporal - IMC e Relação Cintura-Quadril - RCQ). Logo após foi realizada a aferição da PA conforme as recomendações do Ministério da Saúde - MS). ${ }^{1}$

Todas essas etapas foram executadas em salas individualizadas, respeitando-se a privacidade dos sujeitos e propiciando a criação de ambiente organizado e tranquilo de forma a estimular a concentração dos sujeitos. No caso do sujeito da pesquisa ser analfabeto ou não conseguir ler o questionário, a pesquisadora realizou a leitura das perguntas e procedeu a anotação das respostas.

Os dados foram processados em arquivos digitalizados por meio do software Statistical Package for the Social Sciences - SPSS ${ }^{\circledR}$ for Windows versão 20.0, que viabilizou a construção de tabelas para subsidiar a análise das informações de acordo com o objetivo proposto. As questões do questionário MINICHAL foram subdivididas em dois domínios: 1-9 foram agrupadas no domínio estado mental e as questões de 10-16 foram referentes ao domínio manifestações somáticas. Para análise foram empregadas medidas da estatística descritiva como Média Aritmética Ponderada - MAP, Desvio Padrão - DP da média e Mediana (Me), assim o resultado é expresso em escores médios de QV para os dois domínios pré-definidos.

Para avaliar os fatores associados à QV entre hipertensos, foi utilizada como variável dependente a questão 17 do MINICHAL que questiona: "Você diria que sua HAS e o tratamento da mesma têm afetado sua QV?". A resposta para essa questão foi dicotomizada em: não interfere (para os sujeitos que responderam que a HAS não tem afetado em nada a sua QV) e interfere negativamente (para aqueles que responderam que a HAS tem sim piorado sua QV em algum nível). 
Para essa análise, as variáveis do estudo foram agrupadas em: perfil socioeconômico e demográfico e perfil clínico. Perfil socioeconômico e demográfico: as variáveis foram assim categorizadas: sexo (feminino e masculino); cor autodeclarada (branco e pardo/negro); local de nascimento (Montes Claros e outra cidade de MG); idade (anos) ( $\leq 60$ e >60); profissão (população economicamente ativa (PEA) e população não economicamente ativa (PNEA)); escolaridade (analfabeto, $1^{\circ}$ grau e $2^{\circ}$ grau); estado civil (solteiro, casado/união estável, viúvo e divorciado); mora com (própria família e outra situação); reside em (casa própria e outra situação); renda familiar ( $\leq 2$ salários mínimos (SM) e >2 SM). Perfil clínico: as variáveis foram assim dicotomizadas: PA (ótima/limítrofe e alterado); RCQ (adequado e não adequado); IMC (adequado e sobrepeso/obesidade); regula o sal (sim e não); ingere bebida alcoólica (sim e não); fuma (sim e não); pratica atividade física (sim e não); toma medicação corretamente (sim e não); controla os níveis de PA (sim e não); apresenta DCV associada (não e sim).

A verificação da existência de associação entre a variável dependente e as variáveis do perfil socioeconômico, demográfico e clínico foram identificadas pela análise bivariada empregando o teste de qui-quadrado de Pearson, adotando-se um nível de significância de $p \leq 0,05$. O projeto de pesquisa foi encaminhado à Secretaria Municipal de Saúde de Montes Claros na qual a mesma autoriza a realização da pesquisa por meio da assinatura do Termo de Concordância Institucional - TCI.

Este estudo obedeceu aos preceitos éticos estabelecidos pela Resolução $n^{\circ}$ 466/2012, do Conselho Nacional de Saúde - CNS, na qual regulamenta pesquisas envolvendo seres humanos. ${ }^{8}$ O projeto de pesquisa foi apreciado e aprovado pelo Comitê de Ética em Pesquisa da Universidade Estadual de Montes Claros - CEP-UNIMONTES, via Plataforma Brasil, sob parecer consubstanciado $n^{\circ} 311.654 / 2013$, Certificado de Apresentação para Apreciação Ética - CAAE nº 16967013.0.0000.5146.

Os participantes foram orientados quanto às diretrizes do estudo e assinaram o Termo de Consentimento Livre e Esclarecido - TCLE de modo a autorizar a pesquisa. 


\section{Resultados}

ISSN 2179-6750

Dos hipertensos pesquisados, a maior parte pertence ao sexo feminino $(71,1 \%)$; com idade $\geq 66$ anos (50,2\%); de cor parda ou negra (71,1\%); casado (43,5\%); a maioria é de outras cidades de MG (53,8\%); frequentaram até o $1^{\circ}$ grau ou ensino fundamental $(47,8 \%)$; em relação à profissão, a maioria se declarou como não economicamente ativa (70\%); residem em casa própria (81,4\%); moram com a própria família $(85 \%)$; e renda familiar de até dois salários mínimos (77,9\%) (Tabela 1).

Tabela 1. Perfil socioeconômico e demográfico dos hipertensos. Montes Claros, 2013

\begin{tabular}{|c|c|c|c|}
\hline Variáveis & Descrição & $n^{*}$ & $\%$ \\
\hline \multirow{2}{*}{ Sexo } & Feminino & 180 & 71,1 \\
\hline & Masculino & 73 & 28,9 \\
\hline \multirow{2}{*}{ Cor/Raça } & Branco & 73 & 28,9 \\
\hline & Pardo/Negro & 180 & 71,1 \\
\hline \multirow{2}{*}{ Naturalidade } & Montes Claros & 117 & 46,2 \\
\hline & Outra cidade de MG & 136 & 53,8 \\
\hline \multirow{2}{*}{ Idade (anos) } & $\leq 60$ & 126 & 49,8 \\
\hline & $>60$ & 127 & 50,2 \\
\hline \multirow{2}{*}{ Profissão } & PEA & 76 & 30 \\
\hline & PNEA & 177 & 70 \\
\hline \multirow{3}{*}{ Escolaridade } & Analfabeto & 33 & 13 \\
\hline & $1^{\circ} \mathrm{grau}$ & 121 & 47,8 \\
\hline & $2^{\circ} \mathrm{grau}$ & 99 & 39,2 \\
\hline \multirow{4}{*}{ Estado civil } & Solteiro & 40 & 15,8 \\
\hline & $\begin{array}{l}\text { Casado(a)/União } \\
\text { estável }\end{array}$ & 110 & 43,5 \\
\hline & Viúvo & 82 & 32,4 \\
\hline & Divorciado & 21 & 8,3 \\
\hline \multirow{2}{*}{ Mora com } & Própria família & 215 & 85 \\
\hline & Outra situação & 38 & 15 \\
\hline \multirow{2}{*}{ Reside em } & Casa própria & 209 & 81,4 \\
\hline & Outra situação & 47 & 18,6 \\
\hline \multirow{2}{*}{ Renda } & Até 2 SM & 197 & 77,9 \\
\hline & $>2 \mathrm{SM}$ & 56 & 22,1 \\
\hline
\end{tabular}

Fonte: Elaborado pelos autores (2013). ${ }^{*} n=253$

No que se refere aos aspectos clínicos da HAS, observou-se que a maior parte dos 
hipertensos apresentou PA ótima (28,1\%); em relação à classificação da PA dicotomizada, a maior parte dos hipertensos apresentou PA entre ótima e limítrofe (65,6\%); observou-se alteração da relação quadril-cintura (RCQ) em 83\% dos hipertensos; houve prevalência de sobrepeso e obesidade quanto à avaliação do índice de massa corporal (IMC) em $66 \%$ da amostra (Tabela 2).

Tabela 2. Perfil clínico dos hipertensos. Montes Claros, 2013

\begin{tabular}{|c|c|c|c|}
\hline Variáveis & Descrição & $\mathbf{n}^{*}$ & $\%$ \\
\hline \multirow{7}{*}{ Classificação da PA } & Ótima & 71 & 28,1 \\
\hline & Normal & 62 & 24,5 \\
\hline & Limítrofe & 33 & 13 \\
\hline & HAS 1 & 41 & 16,2 \\
\hline & HAS 2 & 6 & 2,4 \\
\hline & HAS 3 & 1 & 0,4 \\
\hline & PAS isolada & 39 & 15,4 \\
\hline \multirow{2}{*}{ PA } & Ótima/Limítrofe & 166 & 65,6 \\
\hline & Alterado & 87 & 34,4 \\
\hline \multirow{2}{*}{ RCQ } & Adequado & 43 & 17 \\
\hline & Não adequado & 210 & 83 \\
\hline \multirow{2}{*}{ IMC } & Adequado & 86 & 34 \\
\hline & Sobrepeso/Obesidade & 167 & 66 \\
\hline
\end{tabular}

Fonte: Elaborado pelos autores (2013). ${ }^{*} \mathrm{n}=253$

Quanto às alterações no estilo de vida, a maior parte relatou consumo regular de sal na alimentação (76,3\%); não fazem uso de bebida alcoólica (81\%); não praticam atividades físicas (68,8\%); fazem uso correto da medicação (85\%) conforme prescrição médica; fazem controle da HAS através da aferição da PA na UBS (73,9\%); não são fumantes (85\%); quanto às complicações; apenas 20,2\% informou apresentar DCV associada (Tabela 3). 
Tabela 3. Perfil de hábitos de vida saudável autorreferidos pelos hipertensos. Montes Claros, 2013

\begin{tabular}{|c|c|c|c|}
\hline Variáveis & Descrição & $n^{*}$ & $\%$ \\
\hline \multirow{2}{*}{ Regula o sal } & Sim & 193 & 76,3 \\
\hline & Não & 60 & 23,7 \\
\hline \multirow{2}{*}{ Ingere bebida alcoólica } & Sim & 48 & 19 \\
\hline & Não & 205 & 81 \\
\hline \multirow{2}{*}{ Pratica atividade física } & $\operatorname{Sim}$ & 79 & 31,2 \\
\hline & Não & 174 & 68,8 \\
\hline \multirow{2}{*}{ Toma medicação corretamente } & Sim & 215 & 85 \\
\hline & Não & 38 & 15 \\
\hline \multirow{2}{*}{ Controla os níveis de PA } & Sim & 187 & 73,9 \\
\hline & Não & 66 & 26,1 \\
\hline \multirow{2}{*}{ Fuma } & Sim & 38 & 15 \\
\hline & Não & 215 & 85 \\
\hline \multirow{2}{*}{ Apresenta DCV associada } & Sim & 51 & 20,2 \\
\hline & Não & 202 & 79,8 \\
\hline
\end{tabular}

Fonte: Elaborado pelos autores (2013). ${ }^{\star} n=253$

As perguntas do questionário MINICHAL foram subdivididas em dois domínios, sendo estes: Estado Mental e Manifestações Somáticas, e foi apresentado com medidas descritivas da média aritmética ponderada (MAP), desvio padrão (DP) e mediana (Me). Na análise do MINICHAL, quanto mais próximos de zero forem os escores, melhor a QV apresentada pelos hipertensos. Os resultados mostraram que houve maior comprometimento no domínio Estado Mental (pontuação máxima = 27 pontos): MAP=4,6, $\mathrm{DP}=1,3$ e Me=4,0. No domínio Manifestações Somáticas (pontuação máxima = 21 pontos): $\mathrm{MAP}=3,5, \mathrm{DP}=1,0$ e $\mathrm{Me}=3,3$. Quanto ao escore total (pontuação máxima $=48$ pontos), a MAP geral de QV apresentada foi de 26,1, DP=6,8 e Me=24. Assim, pode-se afirmar que na população estudada não há grande prejuízo da QV (Tabela 4).

Tabela 4. Medidas descritivas do MINICHAL. Montes Claros, 2013

$\begin{array}{cllll}\text { Instrumento de QV } & & \text { MAP } & \text { DP } & \text { Me } \\ \text { MINICHAL } & \text { Estado mental } & 4,6 & 1,3 & 4 \\ & \text { Manifestações somáticas } & 3,5 & 1 & 3,3 \\ & \text { Escore total } & 26,1 & 6,8 & 24\end{array}$

Fonte: Elaborado pelos autores (2013). $n=253$ 
A avaliação da última pergunta do instrumento MINICHAL: 'Você diria que sua HAS e o tratamento da mesma têm afetado sua QV?' indicou que $64,8 \%$ consideram que a HAS e o tratamento para a mesma não afetam sua QV, enquanto $35,2 \%$ responderam afetar de alguma forma.

Tabela 5. Percepção da influência da HAS e de seu tratamento na QV. Montes Claros, 2013

\begin{tabular}{llcc}
\multicolumn{1}{c}{ Variável } & Descrição & $\mathbf{n}^{*}$ & $\%$ \\
\hline \multirow{2}{*}{ Você diria que sua HAS e o tratamento } & Não, absolutamente. & 164 & 64,8 \\
da mesma têm afetado sua QV? & Sim, um pouco. & 58 & 22,9 \\
& Sim, muito. & 15 & 5,9 \\
& & 16 & 6,3
\end{tabular}

Fonte: Elaborado pelos autores (2013). ${ }^{*} \mathrm{n}=253$

A Tabela 6 demonstra a análise bivariada entre variáveis do perfil socioeconômico e demográfico e o impacto da HAS na QV. Neste estudo, as variáveis não interferiram na QV dos hipertensos.

Tabela 6. Análise bivariada entre variáveis do perfil socioeconômico e demográfico e o impacto da HAS na QV. Montes Claros, 2013

\begin{tabular}{|c|c|c|c|c|c|c|}
\hline \multirow[t]{2}{*}{ Variáveis } & \multirow[t]{2}{*}{ Descrição } & \multicolumn{2}{|c|}{ Não interfere } & \multicolumn{2}{|c|}{$\begin{array}{c}\text { Interfere } \\
\text { negativamente }\end{array}$} & \multirow[t]{2}{*}{ Valor $p$} \\
\hline & & $n^{*}$ & $\%$ & $n^{*}$ & $\%$ & \\
\hline \multirow{2}{*}{ Sexo } & Feminino & 118 & 72,0 & 62 & 69,7 & \multirow{2}{*}{0,14} \\
\hline & Masculino & 46 & 28,0 & 27 & 30,3 & \\
\hline \multirow{2}{*}{ Cor/Raça } & Branco & 54 & 32,9 & 19 & 21,3 & \multirow{2}{*}{3,76} \\
\hline & Pardo/Negro & 110 & 67,1 & 70 & 78,7 & \\
\hline \multirow{2}{*}{ Naturalidade } & Montes Claros & 73 & 44,5 & 44 & 49,4 & \multirow{2}{*}{0,56} \\
\hline & Outra cidade de MG & 91 & 55,5 & 45 & 50,6 & \\
\hline \multirow{2}{*}{ Idade (anos) } & $\leq 60$ & 84 & 51,2 & 42 & 47,8 & \multirow[b]{2}{*}{0,37} \\
\hline & $>60$ & 80 & 48,8 & 47 & 52,2 & \\
\hline \multirow{2}{*}{ Profissão } & PEA & 53 & 32,3 & 23 & 25,8 & \multirow{2}{*}{1,15} \\
\hline & PNEA & 111 & 67,7 & 66 & 74,2 & \\
\hline \multirow{2}{*}{ Escolaridade } & Analfabeto & 18 & 11 & 15 & 16,9 & \multirow{2}{*}{1,75} \\
\hline & $1^{\circ} / 2^{\circ} \mathrm{Grau}$ & 146 & 89 & 74 & 83,1 & \\
\hline \multirow{2}{*}{ Estado civil } & Casado/União estável & 70 & 42,7 & 40 & 44,9 & \multirow{2}{*}{0,12} \\
\hline & Viúvo/Divorciado & 94 & 37,3 & 49 & 55,1 & \\
\hline \multirow{2}{*}{ Mora com } & Própria família & 137 & 83,5 & 78 & 87,6 & \multirow{2}{*}{0,76} \\
\hline & Outra situação & 27 & 16,5 & 11 & 12,4 & \\
\hline \multirow{2}{*}{ Reside em } & Casa própria & 136 & 82,9 & 70 & 78,7 & \multirow{2}{*}{0,69} \\
\hline & Outra situação & 28 & 17,1 & 19 & 21,3 & \\
\hline
\end{tabular}


Renda

$$
\leq 2 \mathrm{SM}
$$

119

$>2 \mathrm{SM}$

45

45

72,6

27,4

78

11

ISSN 2179-6750

Fonte: Elaborado pelos autores (2013). ${ }^{\star} n=253$

A Tabela 7 descreve a análise bivariada entre as variáveis do perfil clínico e o impacto da HAS na QV. As variáveis não interferiram na QV dos hipertensos. Porém, na variável sal evidenciou-se que a maioria reduziu o consumo do mesmo, determinando o valor de p estatisticamente significativo, como 0,05 , demonstrando que influenciou na QV, ou seja, para a população estudada, a restrição do sal na dieta representa um impacto negativo da QV.

Tabela 7. Análise bivariada entre variáveis do perfil clínico e o impacto da HAS na QV. Montes Claros, 2013

\begin{tabular}{|c|c|c|c|c|c|c|}
\hline Variáveis & Descrição & Não i & fere & Int & $\begin{array}{l}\text { re } \\
\text { Iente }\end{array}$ & Valor $p$ \\
\hline & & $n^{*}$ & $\%$ & $n^{*}$ & $\%$ & \\
\hline$D_{0}$ & Ótima/Limítrofe & 78 & 47,6 & 28 & 37,5 & \\
\hline$P A$ & Alterada & 86 & 52,4 & 61 & 68,5 & 0,14 \\
\hline DCO & Adequado & 29 & 17,7 & 14 & 15,7 & 15 \\
\hline re & Não adequado & 135 & 82,3 & 75 & 84,3 & 0,15 \\
\hline IMC & Adequado & 9 & 36 & 27 & 30,3 & 01 \\
\hline & Sobrepeso/Obesidade & 105 & 64 & 62 & 69,7 & \\
\hline Rogula ocal & Sim & 126 & 76,8 & 67 & 75,3 & \\
\hline NeEपाa sai & Não & 38 & 23,2 & 22 & 24,7 & 0,0 \\
\hline Ingero hohida alcoólica & Sim & 33 & 20,1 & 15 & 16,9 & 01 \\
\hline ingere pepida alcoolica & Não & 131 & 78,9 & 74 & 81,1 & 0,4 \\
\hline & Sim & 60 & 36,6 & 19 & 21,3 & \\
\hline Pratica atividade tisica & Não & 104 & 63,4 & 70 & 78,7 & 6,23 \\
\hline Toma medicação & Sim & 137 & 83,5 & 78 & 87,6 & 076 \\
\hline corretamente & Não & 27 & 16,5 & 11 & 12,4 & 0,10 \\
\hline Controla os níveic de PA & Sim & 125 & 76,2 & 62 & 69,7 & 128 \\
\hline controla os nivels de PA & Não & 39 & 23,8 & 27 & 30,3 & 1,28 \\
\hline Fuma & Sim & 23 & 14 & 15 & 16,9 & 036 \\
\hline & Não & 141 & 86 & 74 & 83,1 & \\
\hline $\mathrm{D}$ & Sim & 29 & 17,7 & 22 & 24,7 & 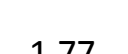 \\
\hline Lev dssucladue & Não & 135 & 82,3 & 67 & 75,3 & $1,1 /$ \\
\hline
\end{tabular}

Fonte: Elaborado pelos autores (2013). ${ }^{*} \mathrm{n}=253$ 


\section{Discussão}

ISSN 2179-6750

A HAS atinge um número cada vez maior de pessoas em todo o mundo. No Brasil, inquéritos de base populacional, realizados em localidades do Sudeste e do Sul, apontaram uma prevalência de HAS de 22,3 a 43,9\%. ${ }^{3}$ Como destacado anteriormente, a HAS é uma doença crônica de evolução lenta, com multiplicidade de fatores etiológicos, tendo como principal agravo o risco de DCV, cerebrovascular e renal que ocasionam elevado ônus pessoal e para o Sistema de Saúde. ${ }^{1}$ Ônus este, com consequências deletérias como internações e procedimentos técnicos de alta complexidade, absenteísmo ao trabalho, óbitos e aposentadorias precoces e comprometimento da QV dos portadores principalmente para àqueles pertencentes aos grupos sociais mais vulneráveis. ${ }^{9}$

A QV tem sido muito abordada em diversas áreas do conhecimento, em especial na área da saúde, pois através dela é possível avaliar a qualidade do cuidado prestado aos portadores de HAS. $^{7}$ A aplicabilidade e o seguimento dos hipertensos quanto ao tratamento proposto é alvo constante de estudos e este fato tem proporcionado aos estudiosos o desenvolvimento de instrumentos cada vez mais precisos para se medir a QV destes indivíduos. Com o uso destes instrumentos é possível entender melhor a doença e propor as melhores formas de intervenção na busca por uma melhor QV. ${ }^{6}$

Em relação ao sexo, esta pesquisa demonstrou que a maior parte da população estudada pertence ao sexo feminino. A predominância de mulheres hipertensas em relação a homens hipertensos já havia sido apontada por vários estudos desenvolvidos no Brasil ${ }^{10}$ Este fato é explicado pela literatura mundial por meio da maior expectativa de vida ao nascer para as mulheres, assim como, por uma busca maior destas pelos serviços de saúde. É importante observar que apesar de haver uma maior preocupação das mulheres com questões relacionadas à saúde não significa necessariamente que estas desfrutem obrigatoriamente de melhores condições de saúde. ${ }^{11}$

Este estudo mostrou que a maioria dos hipertensos se encontra com idade superior a 60 anos. Esse dado pode ser corroborado por outro estudo na qual refere que a HAS é 
mais prevalente na população idosa. ${ }^{2}$ Este resultado se assemelha também a outros estudos encontrados nos quais a maioria dos hipertensos estudados também se encontrava nesta mesma faixa etária. ${ }^{12,13}$

No que se refere à cor autodeclarada, os resultados encontrados neste estudo se assemelham a estudos realizados em todas as capitais brasileiras e no Distrito Federal os quais apontam que a HAS é uma doença prevalente em indivíduos de cor não branca. 2,13,14 A Sociedade Brasileira de Cardiologia - SBC aborda que a HAS é duas vezes mais prevalente em indivíduos de cor não branca. Estudos brasileiros com abordagem simultânea de gênero e cor demonstraram predomínio de mulheres negras com excesso de HAS de até $130 \%$ em relação às brancas, afirma ainda, que não se conhece, com exatidão, o impacto da miscigenação sobre a HAS no Brasil. ${ }^{3}$

Em relação à naturalidade, predominou os entrevistados que informaram ter nascido em outras cidades de MG. A chegada da ferrovia em 1926 em Montes Claros e a transformação da cidade em centro comercial e posteriormente a sua inserção na área de atuação da Superintendência de Desenvolvimento do Nordeste (SUDENE), em 1963, a qual canalizou recursos para a industrialização e a implantação do distrito industrial, serviram como marcos que contribuíram para crescimento da cidade e consequentemente incentivou os processos migratórios. A sua posição como cidade média, que atrai pessoas oriundas, principalmente, do norte de $M G$, é um fato incontestável em termos migratórios positivos dos Censos Demográficos de 1980 a 2010. Desde o ano de 2000, além dos empregos industriais, Montes Claros se destaca pela oferta de ensino superior e pelas possibilidades de se conseguir trabalho no setor terciário, características que reforçam sua posição de macrorregião, principalmente, em relação à assistência à saúde. ${ }^{15}$

É necessário ressaltar que a baixa escolaridade constitui fator de risco para o controle da HAS uma vez que contribui para uma maior dificuldade de entendimento das orientações e aquisição de hábitos de vida saudáveis. No presente estudo, observou-se que grande parte dos sujeitos estudou até o ensino médio, o que pode ser corroborado 
em outro estudo na qual demonstrou uma predominância de indivíduos com HAS que estudaram até o ensino médio e este dado se relacionou com uma maior adesão ao tratamento devido ao fato dos hipertensos entenderem as orientações feitas pela equipe de saúde. ${ }^{16}$

O estado civil que mais prevaleceu foi casado/união estável. Indivíduos que vivem uma relação matrimonial quase sempre recebem mais cuidados do que os viúvos e os solteiros e podem ter mais condições para cuidar do outro e sentirem-se assistidos. ${ }^{17} \mathrm{~A}$ QV, muitas das vezes, está associada à satisfação social e as condições econômicas. Pesquisas apontam que a presença de uma relação familiar estável e uma situação financeira satisfatória leva as pessoas a cuidarem melhor da sua própria saúde, o que faz do apoio familiar um fator facilitador para o tratamento. ${ }^{18}$ Nesta pesquisa identificou-se que a maioria dos participantes respondeu morar com a própria família e residir em casa própria.

Em relação à renda familiar, este estudo demonstrou que a renda mensal na sua maioria é de até dois salários mínimos. Este mesmo dado também foi encontrado na literatura científica na qual demonstrara que recursos financeiros limitados podem levar a uma menor adesão ao tratamento medicamentoso. ${ }^{19}$ Observou-se ainda que a maioria dos sujeitos deste estudo não é economicamente ativa, dependendo da aposentadoria ou outros benefícios para a sua sobrevivência o que os leva a dependerem dos serviços públicos de assistência à saúde.

No que se refere aos valores pressóricos, mais da metade dos hipertensos foi classificado nos estágios ótimo, normal e/ou limítrofe. ${ }^{20}$ Apenas uma minoria dos indivíduos apresentou níveis pressóricos fora da meta estabelecida pelo MS. A grande maioria informou ainda fazer uso correto das medicações. Os valores controlados de PA estão relacionados com o uso correto da medicação, no horário certo, e pelo cuidado do indivíduo em fazer o acompanhamento através da aferição da pressão. ${ }^{21,22}$ Este dado 
reforça que o nível de escolaridade facilita a adesão a tratamentos e, por conseguinte melhora a QV.

A terapêutica para ser efetiva, deve apresentar pouco ou nenhum efeito colateral na vida do cliente. Medicamentos cada vez mais modernos e precisos, com pouco ou nenhum efeito colateral facilitam a adesão dos usuários, pois melhoram a QV. O baixo custo e a facilidade na obtenção da medicação também são essenciais para que haja adesão ao tratamento, isto evita interrupções do controle da pressão e possíveis complicações. ${ }^{2,21,22}$

A maioria dos sujeitos apresentou valores alterados de IMC e RCQ. Em relação à RCQ a maioria apresentou não adequada e o IMC grande parte foi classificado como sobrepeso e obesidade. Este dado está em conformidade com uma pesquisa publicada em 2013 na qual observou que grande parte dos sujeitos foi classificada como obesos, o autor ainda aponta que a obesidade é considerada um importante fator de risco para complicações como o infarto agudo do miocárdio e é causa básica para o Diabetes Mellitus (DM) do tipo II. $^{13,14}$ É importante que os hipertensos façam uso de uma dieta saudável. Pesquisas clínicas e experimentais confirmam a ligação entre obesidade e HAS, obtendo como resultado a constatação de que a presença conjunta das duas condições aumenta consideravelmente o risco cardiovascular, além de tornar o tratamento da HAS ainda mais difícil. ${ }^{23-25}$

Em relação à prática de atividade física, neste estudo a maioria dos sujeitos referiu que não realizam nenhum tipo atividade. Estes dados podem ser confirmados por um estudioso na qual mostra uma predominância de níveis baixo e moderado de atividade física na população, demonstrando um estilo de vida sedentário. ${ }^{26}$ Conforme a SBC, a não adesão ao exercício físico apresenta como um dos principais fatores de risco para as DCV. ${ }^{3}$

A SBC destaca ainda a importância da prática de atividade física para melhorar a QV, esta deve ser realizada por pelo menos 30 minutos ao dia, durante cinco dias por semana. ${ }^{3}$ Podem ser realizadas a caminhada e os exercícios aeróbios que devem ser 
complementados pelos resistidos, estes promovem reduções de PA, estando indicados com o intuito de prevenir e tratar a HAS e os riscos cardiovasculares.

Em pessoas com consumo reduzido de sal é observada menor prevalência de HAS, sendo que a PA não se eleva com a idade. A relação entre aumento da PA e o avanço da idade é maior em populações com alta ingestão de sal. ${ }^{23}$ Nesta pesquisa o consumo de sal foi referido pelos sujeitos como adequado ao informarem que reduziram o consumo do mesmo após o diagnóstico de HA. Este dado difere de outra pesquisa encontrada na qual aponta a necessidade eminente de alteração no padrão de consumo de sal. ${ }^{24}$ Quanto à significância da análise bivariada empregando o teste de qui-quadrado de Pearson, o sal apresentou-se como a única variável com correlação com a QV dos hipertensos. Isso se explica porque quando há a redução da ingestão de sal há queda nos valores pressóricos, menor ocorrência de complicações e consequentemente melhora na QV.

O consumo crônico de dieta com conteúdo elevado de sal está associado com aumento da PA e maior mortalidade por DCV. A ingestão de sal está diretamente relacionada às alterações da PA e às complicações cardiovasculares, demonstrando a relevância do controle dietético, principalmente dos alimentos ricos em sal, como os enlatados e refrigerantes. ${ }^{1}$

Divergentes respostas pressóricas às variações na ingestão de sódio são identificadas em indivíduos e as causas para tal heterogeneidade não estão completamente explicadas. A maioria dos estudos analisados mostra efeitos benéficos da restrição moderada de sódio da dieta, associada ou não a outras modificações nutricionais ou ao aumento da atividade física, tanto em parâmetros metabólicos quanto na PA. Em suma, a resposta da PA diante do sal é influenciada pela condição, possivelmente geneticamente determinada de sensibilidade ao sal, mas ainda não está claro como isso interfere no controle da PA das pessoas. ${ }^{23,24}$

Embora, o efeito do sal sobre os níveis pressóricos parece ser heterogêneo. Pesquisas apontam que indivíduos podem apresentar distintas respostas fisiológicas à 
ingestão de sódio, ou seja, sensibilidade ao sal. Enquanto algumas pessoas apresentam um anormal aumento da PA em resposta ao aumento da ingestão de sal, denominadas de salsensíveis, outras podem apresentar pequenas alterações nos níveis pressóricos mesmo consumindo grandes quantidades de sal, sendo chamadas de sal-resistentes. Tais indícios intensificam a necessidade de informação aos hipertensos e pré-hipertensos quanto aos benefícios da diminuição de sódio na alimentação. ${ }^{22-26}$

A ingestão de sal excede os limites máximos recomendados em todos os países, em todas as condições de renda. É evidenciado que a restrição de sal acompanhada de hábitos alimentares saudáveis contribui para a redução da PA, podendo levar à diminuição da medicação anti-hipertensiva e consequente melhora no estado de saúde e QV. ${ }^{19,23,24}$

Em relação ao hábito de fumar, os resultados desta pesquisa foram idênticos a um estudo realizado no interior de São Paulo (SP). ${ }^{26}$ Estes demonstraram redução do hábito de fumar entre hipertensos nos últimos anos. Conforme a $\mathrm{SBC}^{3}{ }^{3}$ o hábito de fumar apresenta como um dos principais fatores de risco para as DCV, a cessação do tabagismo constitui medida fundamental e prioritária na prevenção primária e secundária das doenças do coração, bem como de tantas outras, a exemplo o câncer de pulmão.

No presente estudo, observou-se que um pequeno número de pessoas relatou ingerir bebida alcoólica, o que difere de uma constatação científica na qual nenhum dos sujeitos realizava controle da ingesta de álcool. ${ }^{27}$ Foi detectado um importante consumo de bebidas alcoólicas, principalmente entre sujeitos do sexo masculino, o resultado encontrado pode estar relacionado ao maior número de mulheres na amostra estudada já que estudos apontam maior aderência das mulheres aos tratamentos. ${ }^{28}$

Observou-se que uma minoria dos pesquisados relatou apresentar DCV associada. A HAS é uma doença comum, que pode permanecer durante um longo período de tempo sem sintomas e é considerado um dos principais fatores de risco para as DCV e AVC, por si só, mas largamente potenciados se estiverem presentes outros fatores como o tabaco, excesso de peso, sedentarismo ou $\mathrm{DM}^{29}$ Indivíduos com HAS têm três vezes maior 
probabilidade de desenvolver doença cardíaca ou AVC, e duas vezes maior probabilidade de virem a morrer destas doenças, quando comparados a indivíduos com valores pressóricos normais. ${ }^{1,11-13}$

Quando questionadas sobre a presença de história familiar de HAS, a grande maioria informou apresentar casos na família. A contribuição de fatores genéticos para a determinação da HAS encontra-se bem determinada na população. ${ }^{3}$ As pesquisas apontam que o melhor controle da PA tem um impacto positivo na QV, sendo um fator independente para a melhoria da mesma. Este fato acontece devido ao não controle da PA trazer uma maior reação de ansiedade e depressão e, consequentemente, uma piora na QV.

Em se tratando da QV de hipertensos, também foi encontrado que quanto melhor a percepção do paciente em relação à sua doença e o respectivo tratamento, melhor também será a sua avaliação nos domínios estado mental e manifestações somáticas. Pessoas que realizam atividade física possuem uma melhor percepção de QV no domínio estado mental, pois sentem que estão possuindo hábitos de vida saudáveis e com isso buscando o controle da HAS e prevenindo contra complicações dessa doença. ${ }^{16}$ Este estudo demonstrou que a condição clínica da HAS não afetou a QV dos sujeitos, eles demonstraram conhecimento acerca de sua situação de saúde bem como entendem sobre a importância do seguimento ao tratamento medicamentoso e não medicamentoso no controle da HAS. É importante ressaltar que os sujeitos deste estudo apresentam diagnóstico médico estabelecido e se encontram em tratamento há mais de seis meses.

\section{Conclusão}

Discutir os principais fatores determinantes das condições e autopercepção da QV da população hipertensa é essencial para conhecer a sua avaliação acerca desse assunto, por ser um fator preponderante para avaliar a melhora do indivíduo após o início do tratamento, bem como identificar as alterações causadas pela doença e pelo seu tratamento no cotidiano. 
Esta pesquisa mostrou que, a população estudada é composta em sua grande maioria por mulheres, com idade superior a 60 anos, de cor predominantemente não branca, sem companheiros, que estudaram até o Ensino Médio, não economicamente ativas, naturais de cidades do norte do Estado de Minas Gerais, incluindo Montes Claros, que residem com a própria família, em casa própria, com renda familiar de até dois salários mínimos.

A maioria apresentou PA ótima e/ou limítrofe, com IMC e RCQ alterados, não fazem uso de bebida alcoólica e nem de cigarros e não realizam atividade física. Reduziram a ingestão de sal após a confirmação da doença, usam os medicamentos hipotensores e controlam os níveis pressóricos de acordo com as orientações médicas e não apresentam DCV associada.

A melhor QV nos indivíduos hipertensos pode estar associada à postura frente à doença e aos cuidados tomados para o controle da PA. Este estudo demonstrou que a percepção da QV não está condicionada a doença, nos domínios do instrumento MINICHAL o estado mental impactou mais sobre a QV do que as manifestações somáticas. Isso pode demonstrar que a QV decorre de ações que causam conforto e segurança tanto psíquicas como físicas.

Os resultados apontam que a QV mensurada, tanto por seus componentes isolados como de uma maneira geral, não se encontra relacionado à doença HAS. Por outro lado, o reconhecimento do risco, ou da gravidade da doença, dado pela quantidade de medicamentos hipertensivos usados e pelo nível de PAS, pode ser utilizado como ferramenta educativa para auxiliar a adoção de um comportamento benéfico no que se refere ao consumo de sal e outros hábitos de vida saudáveis.

A escolha dos participantes desta pesquisa foi realizada conforme o cadastro das pessoas diagnosticadas com HAS na ESF, restringindo os resultados deste estudo à parcela da população hipertensa que conhece o diagnóstico e realiza tratamento. Pesquisas posteriores podem analisar a relação entre QV de hipertensos e normotensos, bem como 
entre hipertensos que aderem ou não ao tratamento, incluindo outras variáveis que podem interferir no controle da PA, como por exemplo: depressão, estresse, presença de patologias associadas e comorbidades, número de medicamentos utilizados, dentre outros para que possa haver mais variáveis que possam indicar se interfere ou não na QV das pessoas com pressão alterada.

Espera-se que este estudo possa contribuir para uma melhor assistência aos hipertensos atendidos neste serviço, aumentando a discussão acerca do tema, privilegiando-o no planejamento das atividades, bem como identificando a percepção dos indivíduos sobre como a HAS influencia na sua QV, por ser tratar de um fator preponderante no controle da doença.

\section{Referências}

1. Brasil. Ministério da Saúde. Estratégias para o cuidado da pessoa com doença crônica: hipertensão arterial sistêmica. Brasília, DF: MS; 2013. (Cadernos de atenção básica, n 37).

2. Silva PLN, Rodrigues GF, Rodrigues GF, Mota EC, Souto SGT, Gonçalves RPF. Avaliação epidemiológica dos hipertensos cadastrados em uma unidade básica de saúde. Rev Enferm UFPE on line. 2014;8(8):2617-25. https://doi.org/10.5205/1981-8963-v8i8a9963p2617-26252014.

3. Sociedade Brasileira de Cardiologia. VI Diretrizes Brasileiras de Hipertensão. Arq Bras Cardiol. 2010 [citado 28 ago. 2019];95(1):1-51. Disponível em:

http://publicacoes.cardiol.br/consenso/2010/Diretriz_hipertensao_associados.pdf.

4. Borges JWP, Pinheiro NMG, Souza ACC. Hipertensão comunicada e hipertensão compreendida: saberes e práticas de enfermagem em um programa de saúde da família de Fortaleza, Ceará. Cienc Saude Colet. 2012;17(1):179-89. http://dx.doi.org/10.1590/S1413-81232012000100020.

5. Oliveira RAR, Mota Júnior RJ, Tavares DDF, Moreira OC, Marins JCB. Fatores associados à pressão arterial elevada em professores da educação básica. Rev Educ Fis UEM. 2015;26(1):11929. http://dx.doi.org/10.4025/reveducfis.v26i1.24693.

6. Povoa TIR, Jardim PCBV, Sousa ALL, Jardim TSV, Souza WKSB, Jardim LSV. Treinamento aeróbio e resistido, qualidade de vida e capacidade funcional de hipertensas. Rev Bras Med Esporte. 2014;20(1):36-41. http://dx.doi.org/10.1590/S1517-86922014000100007.

7. Soutello ALS. Qualidade de vida e hipertensão: avaliação ampliada do desempenho psicométrico da versão brasileira do "Mini-Cuestionario de Calidad de vida en la Hipertensión 
Arterial - Minichal" [dissertação]. Campinas (SP): Universidade Estadual de Campinas, Faculdade de Ciências Médicas; 2010.

8. Brasil, Ministério da Saúde, Conselho Nacional de Saúde. Resolução n 466, de 12 de dezembro de 2012. Aprova as diretrizes e normas regulamentadoras para pesquisas envolvendo seres humanos. Brasília, DF: Diário Oficial da União; 13 jun. 2016.

9. Ribeiro AG, Cotta RMM, Ribeiro SMR, Dias CMGC, Araújo RMA. Representações sociais de mulheres portadoras de hipertensão arterial sobre sua enfermidade: desatando os nós da lacuna da adesão ao tratamento na agenda da Saúde da Família. Physis. 2011;21(1):87-112. http://dx.doi.org/10.1590/S0103-73312011000100006.

10. Corrêa-Neto VG, Sperandei S, Silva LAI, Maranhão-Neto GA, Palma A. Hipertensão arterial em adolescentes do Rio de Janeiro: prevalência e associação com atividade física e obesidade. Cienc Saude Colet. 2014;19(6):1699-708. http://dx.doi.org/10.1590/141381232014196.05262013.

11. Tavares DMS, Paiva MM, Dias FA, Diniz MA, Martins NPF. Características sociodemográficas e qualidade de vida de idosos com hipertensão arterial sistêmica que residem na zona rural: importância do papel do enfermeiro. Rev Latino-Am Enferm. 2013;21(2):515-22. https://doi.org/10.1590/S0104-11692013000200007.

12. Alves MCB, Brito FN, Lima LM, Matos VC, Santos JC, Leite DKM, et al. A qualidade de vida de pacientes hipertensos em uma estratégia saúde da família, Ananindeua - Pará. Rev Eletr Gestao Saude. 2013;4(1):1378-90.

13. Almeida LML, Neves JV, Amaral EO, Silva PLN. Prevalência da hipertensão arterial sistêmica em uma Estratégia Saúde da Família de um município norte mineiro. Rev Eletr Gestao Saude. 2015;6(1):349-65.

14. Carvalho MAN, Silva IBS, Ramos SBP, Coelho LF, Gonçalves ID, Figueiredo Neto JA. Qualidade de vida de pacientes hipertensos e comparação entre dois instrumentos de medida de QVRS. Arq Bras Cardiol. 2012;98(5):442-51. http://dx.doi.org/10.1590/S0066-782X2012005000032.

15. Leal AA. Migração interna em cidades médias: aspectos socioeconômicos e espacialidade dos imigrantes de Montes Claros - MG a partir de análises do Censo Demográfico de 2010 [dissertação]. Montes Claros (MG): Universidade Estadual de Montes Claros; 2013.

16. Andrade SSA, Stopa SR, Brito AS, Chueri PS, Szwarcwald CL, Malta DC. Prevalência de hipertensão arterial autorreferida na população brasileira: análise da Pesquisa Nacional de Saúde, 2013. Epidemiol Serv Saude. 2015;24(2):297-304. http://dx.doi.org/10.5123/S167949742015000200012.

17. Bezerra SMMS, Veiga EV. Qualidade de vida entre pessoas com hipertensão arterial atendidos em unidades de estratégias de saúde da família. Rev Enferm UFPE on line. 2013;7(esp):7055-63. http://dx.doi.org/10.5205/reuol.4767-42136-1-ED.0712esp201313. 
18. Ribeiro ISS, Boery RNO, Casotti CA, Freire IV, Boery EN. Qualidade de vida de hipertensos atendidos na atenção primária à saúde. Saude Debate. 2015;39(105):432-40. http://dx.doi.org/10.1590/0103-110420151050002011.

19. Mian NC, Gasparino RC. Qualidade de vida de hipertensos em tratamento ambulatorial. Cogitare Enferm. 2012;17(3):519-23. http://dx.doi.org/10.5380/ce.v17i3.29294.

20. Nascente FMN, Jardim PCBV, Peixoto MRG, Monego ET, Moreira HG, Vitorino PVO, et al. Hipertensão arterial e sua correlação com alguns fatores de risco em cidade brasileira de pequeno porte. Arq Bras Cardiol. 2010;95(4):502-9. http://dx.doi.org/10.1590/S0066782X2010005000113.

21. Oliveira CJ, Moreira TMM. Caracterização do tratamento não-farmacológico de idosos portadores de hipertensão arterial. Rev Rene. 2010;11(1):76-85.

22. Cavalari E, Nogueira MS, Fava SMCL, Cesarino CB, Martin JFV. Adesão ao tratamento: estudo entre portadores de hipertensão arterial em seguimento ambulatorial. Rev Enferm UERJ. 2012;20(1):67-72.

23. Perin MS, Cornélio ME, Rodrigues RCM, Gallani MCBJ. Caracterização do consumo de sal entre hipertensos segundo fatores sociodemográficos e clínicos. Rev Latino-Am Enferm. 2013;21(5):[09 telas].

24. Costa FP, Machado SH. O consumo de sal e alimentos ricos em sódio pode influenciar na pressão arterial das crianças? Cienc Saude Colet. 2010;15(supl. 1):1383-9.

25. Gomes F, Telo DF, Souza HP, Nicolau JC, Halpern A, Serrano Junior CV. Obesidade e doença arterial coronariana: papel da inflamação vascular. Arq Bras Cardiol. 2010;94(2):255-61. http://dx.doi.org/10.1590/S1413-81232010000700048.

26. Massa KHR, Guimarães VV, César CLG, Barros MBA, Carandina L, Goldbaum M, et al. Prática de atividade física em quatro domínios em idosos com hipertensão arterial referida no Município de São Paulo: estudo de base populacional. Rev Bras Ativ Fis Saude. 2012;17(1):7-13. https://doi.org/10.12820/rbafs.v.17n1p7-13.

27. Helena ETS, Nemes MIB, Eluf-Neto J. Avaliação da assistência a pessoas com hipertensão arterial em Unidades de Estratégia Saúde da Família. Saude Soc. 2010;19(3):614-26. http://dx.doi.org/10.1590/S0104-12902010000300013.

28. Duarte MTC, Cyrino AP, Cerqueira ATAR, Nemes MIB, Iyda M. Motivos do abandono do seguimento médico no cuidado a portadores de hipertensão arterial: a perspectiva do sujeito. Cienc Saude Colet. 2010;15(5):2603-10. http://dx.doi.org/10.1590/S1413-81232010000500034.

29. Mendonça FF, Nunes EFPA. Avaliação de grupos de educação em saúde para pessoas com doenças crônicas. Trab Educ Saude. 2015;13(2):397-409. http://dx.doi.org/10.1590/1981-7746sip00053. 
Patrick Leonardo Nogueira da Silva | ORCiD: 0000-0003-2399-9526

Especialista em Enfermagem do Trabalho pela Faculdade de Guanambi - FG. Professor da Escola Técnica de Saúde do Centro de Educação Profissional e Tecnológica da Universidade Estadual de Montes Claros ETS/CEPT/UNIMONTES. Montes Claros, MG, Brasil.

Daniele Cristina Moreira | ORCiD: 0000-0002-0243-7893

Mestranda pelo Programa de Pós-Graduação em Ciências da Saúde - PPGCS) da Universidade Estadual de Montes Claros - UNIMONTES. Montes Claros, MG, Brasil.

Aurelina Gomes e Martins | ORCiD: 0000-0003-3582-8994

Mestre em Saúde Pública pela Universidade Federal de São Paulo - UNIFESP. Professora do Departamento de Enfermagem da Universidade Estadual de Montes Claros - UNIMONTES. Montes Claros, MG, Brasil.

Elaine Cristina Santos Alves | ORCiD: 0000-0003-1357-9341

Mestre em Ciências da Saúde pela Universidade Federal de São Paulo - UNIFESP. Professora do Denartamento de Fnfermanem da I Inivercidade Fstadıal de Montec Clarnc - I INIMONTFS Mnntec Clarnc 